\title{
Group-Level Emotion Recognition using Hybrid Deep Models based on Faces, Scenes, Skeletons and Visual Attentions
}

\author{
Xin Guo \\ Department of Electrical and \\ Computer Engineering, University of \\ Delaware \\ Newark, DE, USA \\ guoxin@udel.edu
}

\author{
Bin Zhu \\ Department of Electrical and \\ Computer Engineering, University of \\ Delaware \\ Newark, DE, USA \\ zhubin@udel.edu
}

\author{
Luisa F. Polanía \\ American Family Mutual Insurance \\ Company \\ Madison, WI, USA \\ polania@amfam.com
}

\author{
Charles Boncelet \\ Department of Electrical and \\ Computer Engineering, University of \\ Delaware \\ Newark, DE, USA \\ boncelet@udel.edu
}

\author{
Kenneth E. Barner \\ Department of Electrical and \\ Computer Engineering, University of \\ Delaware \\ Newark, DE, USA \\ barner@udel.edu
}

\begin{abstract}
This paper presents a hybrid deep learning network submitted to the 6th Emotion Recognition in the Wild (EmotiW 2018) Grand Challenge [9], in the category of group-level emotion recognition. Advanced deep learning models trained individually on faces, scenes, skeletons and salient regions using visual attention mechanisms are fused to classify the emotion of a group of people in an image as positive, neutral or negative. Experimental results show that the proposed hybrid network achieves $78.98 \%$ and $68.08 \%$ classification accuracy on the validation and testing sets, respectively. These results outperform the baseline of $64 \%$ and $61 \%$, and achieved the first place in the challenge.
\end{abstract}

\section{CCS CONCEPTS}

- Computing methodologies $\rightarrow$ Activity recognition and understanding; Scene understanding; Transfer learning;

\section{KEYWORDS}

EmotiW 2018; Group-level Emotion Recognition; Multi-model; Scene Understanding; Visual Attention;

\section{ACM Reference Format:}

Xin Guo, Bin Zhu, Luisa F. Polanía, Charles Boncelet, and Kenneth E. Barner. 2018. Group-Level Emotion Recognition using Hybrid Deep Models based on Faces, Scenes, Skeletons and Visual Attentions. In 2018 International Conference on Multimodal Interaction (ICMI '18), October 16-20, 2018, Boulder, CO, USA. ACM, New York, NY, USA, 5 pages. https://doi.org/10.1145/3242969. 3264990

Permission to make digital or hard copies of all or part of this work for personal or classroom use is granted without fee provided that copies are not made or distributed for profit or commercial advantage and that copies bear this notice and the full citation on the first page. Copyrights for components of this work owned by others than the author(s) must be honored. Abstracting with credit is permitted. To copy otherwise, or republish, to post on servers or to redistribute to lists, requires prior specific permission and/or a fee. Request permissions from permissions@acm.org.

ICMI '18, October 16-20, 2018, Boulder, CO, USA

(c) 2018 Copyright held by the owner/author(s). Publication rights licensed to ACM. ACM ISBN 978-1-4503-5692-3/18/10...\$15.00

https://doi.org/10.1145/3242969.3264990

\section{INTRODUCTION}

Group-level emotion recognition has been a topic of interest in the social psychology community for decades [24]. The interest in this topic is motivated by the fact that group level emotions are essential in understanding the sense of social identity and how individuals relate to their social environment. Social psychology is not the only area interested in group emotions. This topic also has applications in shot selection [7], image retrieval [6], surveillance [2], event detection [32], and event summarization [7], which motivates the design of automatic systems capable of understanding human manifestations of emotional attributes at the group level. The EmotiW Group-level Emotion Recognition Sub-challenge was created with the aim of advancing group-level emotion recognition. In this annual sub-challenge, the collective emotion is classified as positive, neutral, or negative using the Group Affect Database 2.0 [8].

Prior work in the area includes the work of Dhall et al. [8], which introduces the Group Affect Database and a framework for group emotion recognition which includes the extraction of facial features using Facial Action Units, extraction of low-level features on the aligned faces, extraction of scene features using the GIST and CENTRIST descriptors and fusion using Multiple Kernel Learning. Another work that also uses hand-crafted features is described in [17]. Specifically, the authors used the Riesz transform and the local binary pattern descriptor to exploit not only neighbouring changes in the spatial domain of a face but also along the different Riesz faces.

Different approaches based on deep neural networks have been proposed to solve the task of the Group-level Emotion Recognition Sub-challenge. In 2016, the winner of the sub-challenge proposed the extraction of features from both the whole image and facial regions using the combination of a convolutional neural network (CNN) and a long short-term memory network (LSTM) [19]. Similarly, the winner of the 2017 version of the sub-challenge proposed to fuse the results of CNNs trained individually on faces and whole images [30]. The authors used a large-margin softmax loss for discriminative learning. In [11], we presented a hybrid network that 


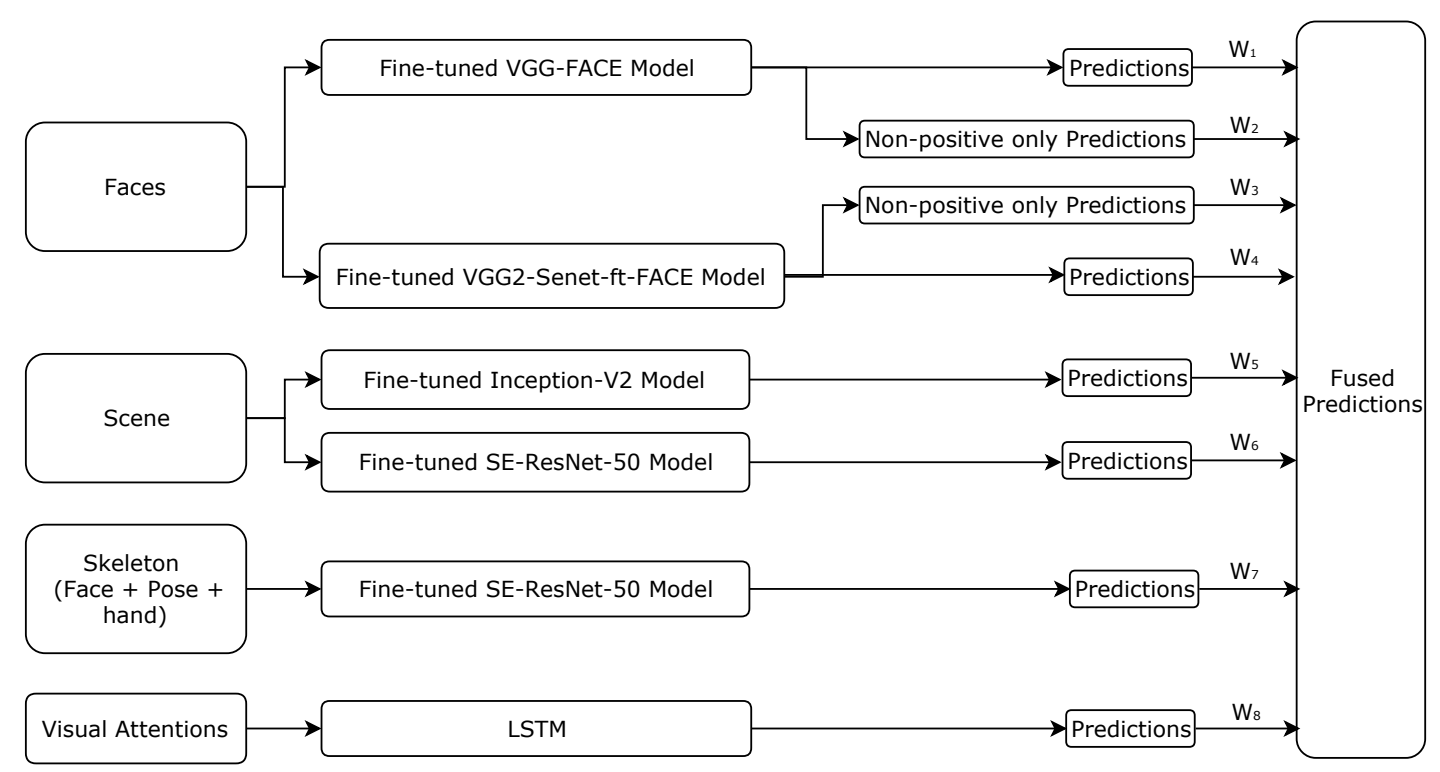

Figure 1: The overall structure of the proposed hybrid network. The network contains 8 deep models trained on scenes, faces, skeletons and visual attentions separately. $\sum_{i} W_{i}=1$, and $0 \leq i \leq 8$. Details are described in Section 2 and 3.

exploited information from whole images, faces and the skeleton representation of the subjects in the image using CNNs.

The problem of group emotion recognition is challenging due to face occlusions, illumination variations, head pose variations, varied indoor and outdoor settings, and faces at different distance from the camera which may lead to low-resolution face images [23]. In this paper, we propose a method to address this challenging problem by using a hybrid network that fuses the predictions of models trained individually on faces, scenes, skeletons and regions extracted with visual attention mechanisms. One of the models is trained on faces because facial expressions convey powerful discriminating information for emotion recognition. The model trained on scenes captures context information while the model trained on skeleton representations captures body gestures, which convey important affective information according to research results on experimental psychology and affective computing [26]. Visual attention is exploited by one of the models to enable deeper image understanding through fine-grained analysis by focusing on regions of the image that are salient and relevant for the emotion recognition task. The proposed hybrid network achieved the first place in the Group-level Emotion Recognition Sub-challenge, reporting a classification accuracy of $78.98 \%$ and $68.08 \%$ on the validation and testing sets, respectively. Code is available at https://github.com/gxstudy/EmotiW2018_Grouplevel_Emotion_Recognition.

\section{THE PROPOSED METHOD}

The general idea of the proposed method is to train multiple deep models to learn high-level abstractions of the input from different perspectives, so that the learned models can complement each other and be fused into a high-performance hybrid network (Figure 1).

\subsection{Face Prediction}

The VGG-FACE model is a 16-layer VGG architecture trained on a large-scale dataset (VGGFace dataset [25]), containing 2.6M images of $2.6 \mathrm{~K}$ celebrities, for face recognition. It has been shown that fine-tuning the VGG-FACE model for the task of facial emotion recognition achieves better results than using hand-engineered features or deep models trained from scratch $[11,12]$.

A new face-related model, VGG2-Senet-ft-FACE, was recently proposed by the VGG group in [3]. VGG2-Senet-ft-FACE is a ResNet50 [14] network with Squeeze-and-Excitation (SE) blocks [16], which was initially pretrained on the Ms-Celeb-1M dataset [13] and finetuned on the VGGFace2 [3] training set. The VGGFace2 dataset contains 3.31 million images of 9131 subjects.

The VGG2-Senet-ft-FACE model and VGG-FACE architectures are modified in this paper to address the problem of group-level emotion recognition. The modification consists of changing the number of neurons in the last fully-connected layer to 3 . The modified architectures are initialized with the weights of the original VGG-FACE and VGG2-Senet-ft-FACE model individually, with the exception of the last fully-connected layer, which is initialized with weights sampled from a Gaussian distribution of zero mean and variance $1 \times 10^{-4}$. The learning parameters of each architecture, such as the overall learning rate, the weight decay, and the learning policy are set the same as in the original models, except that the last fully connect layer is trained with a learning rate for the weight and bias terms that is set to be 10 times larger than the overall learning rate.

Faces are extracted and aligned using MTCNN [39]. The modified VGG-FACE and VGG2-Senet-ft-FACE models are first fine-tuned on a combined facial emotion dataset (30205 samples in total), which includes images from the facial expression recognition 2013 (FER2013) dataset [10] and the GENKI-4K dataset [35]. The negative 
collection is formed with the angry and sad classes from the FER2013 database, the neutral collection is formed by combining neutral images from both datasets, and the positive is formed by combining the happy images from both datasets.

The models are further fine-tuned on the detected faces of the Group Affect Database 2.0. During training, all the extracted faces are re-scaled to $256 \times 256$ pixels and have the same weight when fine-tuning the parameters of the network. For scoring, the mean of the prediction of individual faces is used as the final prediction.

\subsection{Scene Prediction}

Deep models based on whole images (scene) have demonstrated superior performance compared to hand-engineered features, such as CENTRIST [36], in [11, 30]. A new state-of-the-art model, SENet [16], which is the winner of the ImageNet 2017 Large Scale Visual Recognition Challenge (ILSVRC 2017) [5], is based on the idea of stacking Squeeze-and-Excitation (SE) blocks together to improve the representational power of a network by explicitly modeling the interdependencies between the channels of its convolutional features. Squeeze-and-Excitation blocks can be applied to state-of-the-art deep convolutional networks such as AlexNet [18], VGGNet [28] and ResNet [14]. Inception-V2 [29] and SE-ResNet-50, which is a 50-layer ResNet with SE blocks, trained on ImageNet-1K database, are used as scene models in this paper.

Specifically, whole images are used as input to SE-ResNet-50 and Inception-V2 deep networks. Each architecture is modified by changing the number of neurons in the last layer to 3, indicating a ternary classification, having as targets negative, neutral and positive emotions for the group. With the exception of the last layer, the modified architectures are initialized with the models trained on ImageNet. The last layer in each architecture is initialized in the same way as in the original setup of each architecture, and trained with a learning rate for the weight and bias terms that is set to be 10 times larger than the overall learning rate. The learning parameters of each architecture, such as the overall learning rate, the weight decay, and the learning policy are set the same as in the original submissions to the ImageNet challenge.

\subsection{Skeleton Classification}

Skeletons corresponding to the collection of keypoints of human face, body and hands were first used for the problem of groupemotion recognition in [11]. As shown in Figure 2, skeleton features demonstrate salient patterns of different categories through facial expression, people layout, pose and gestures. In this paper, we propose to fine-tune an SE-ResNet-50 model on skeletons. The skeleton of each image is extracted using OpenPose [4, 31, 34], which can jointly detect human body, hand and facial keypoints (130 keypoints in total for each person) on single images, invariant to the number of people in the image. Model modification, training parameters and training procedure are the same as in the SE-ResNet50 model trained on scene images.

\subsection{Visual Attention Classification}

Visual attention mechanisms have been widely explored in image captioning [1,21,27], visual question answering [37, 38] and image classification $[22,33]$. In this paper, we propose to use areas of

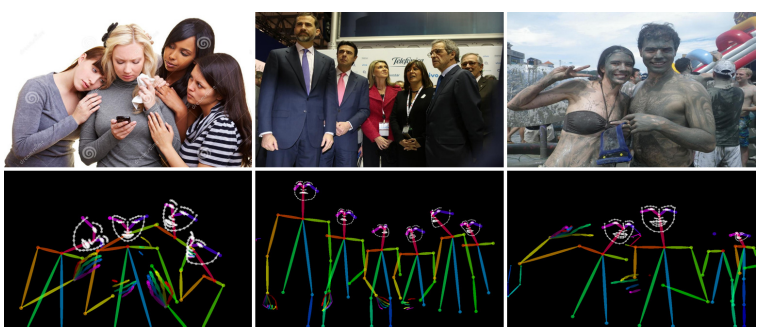

Figure 2: Samples of skeleton representations. Left: original image. Middle: skeleton representation 1 (includes faces and bodies). Right: skeleton representation 2 (includes faces, bodies and hands)

images that contain salient objects or features to classify grouplevel emotions. Specifically, 16 salient regions are extracted using the combined bottom-up and top-down attention mechanism proposed in [1], then a SENet-154 model $^{1}$ trained on the ImageNet-1k database is used to extract a 2048-dimensional vector from each region at layer pool5/7x7_s $1^{2}$. As shown in Figure 3, the attention mechanism is able to detect and crop salient features such as people, white signs, red signs, pink mats and bouquets.

After feature extraction, each image is represented by 16 feature vectors (each vector of dimension $2048 \times 1$ ). Keeping only 16 regions can be viewed as a hard attention mechanism, as only a small number of image regions are selected from a large number of region proposals. A single-layer LSTM [15] with 128 neurons is trained on the extracted attention features. The network takes a sequence of 16 feature vectors and learns to combine features from 16 salient areas and predict the overall emotion of the image. The learning rate is set to 0.001 , the bias terms are initialized to 0 , and the weights of the LSTM are initialized with values drawn from a truncated normal distribution with mean 0 and standard deviation 0.1 . The training process runs for 10 epochs using Adam optimizer.

\section{EXPERIMENTAL RESULTS}

\subsection{Group-Level Emotion Recognition Sub-challenge}

Group-level emotion recognition is one of the sub-challenges in the 6th Emotion Recognition in the Wild (EmotiW 2018) Grand Challenge [9]. The images in this sub-challenge are from the Group Affect Database 2.0 [8], which contains 9815, 4346 and 3011 images in the training, validation and testing sets, respectively ${ }^{3}$. These images are collected from social events, such as convocations, marriages, parties, meetings, funerals, protests, etc. Participants compete on the accuracy of classifying the group perceived emotion as positive, neutral or negative on the testing data.

\subsection{Experimental Details}

The prediction accuracy of the models tested on the validation dataset is shown in Table 1. Large-margin softmax loss [20] is used in each model, with the exception of the attention-LSTM model, to

\footnotetext{
${ }^{1}$ Model downloaded from https://github.com/hujie-frank/SENet

${ }^{2}$ pool5/7x7_s1 is a layer name

${ }^{3}$ Note that in 2017 the numbers of images were 311, 165, and 296, respectively
} 

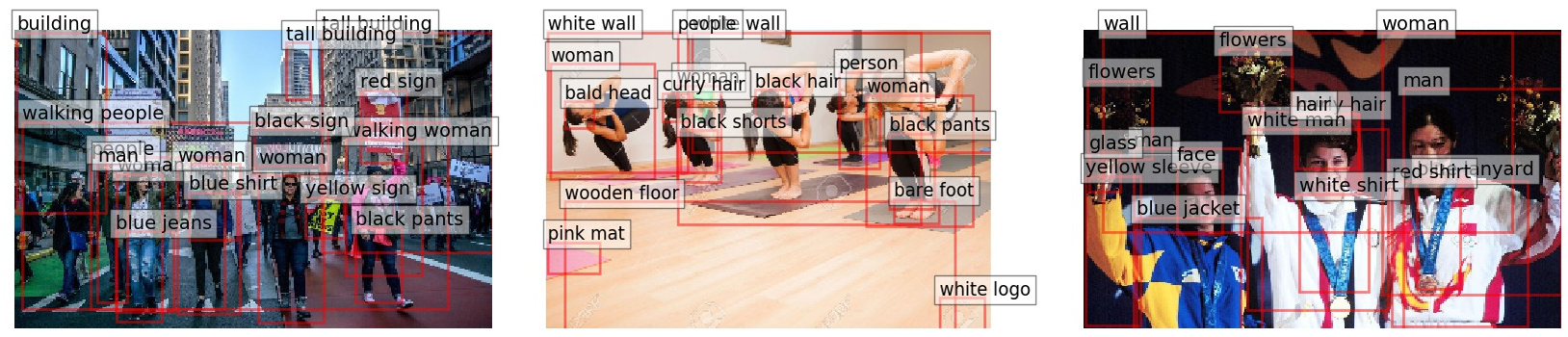

Figure 3: Examples of visual attentions. Left: negative. Middle: neutral. Right: Positive.

Table 1: Performance of each model on the validation set.

\begin{tabular}{|c|c|c|}
\hline Models & Acc & +LargeMargin \\
\hline VGG-FACE Model & 68.28 & 70.07 \\
\hline VGG2-Senet-ft-Face Model & 68.94 & 70.55 \\
\hline Inception-V2 Scene Model & 67.21 & 68.09 \\
\hline SE-ResNet-50 Scene Model & 68.16 & 68.38 \\
\hline SE-ResNet-50 Skeleton Model & 64.42 & 65.87 \\
\hline Attention LSTM Model & 67.46 & - \\
\hline
\end{tabular}

Table 2: Accuracies of model fusions on the validation set.

\begin{tabular}{|c|c|}
\hline Fused Models & Acc \\
\hline VGG-FACE Model + VGG-FACE Non-positive & 71.79 \\
\hline + VGG2-Senet-ft-Face Model & 73.49 \\
\hline +VGG2-Senet-ft-Face Model Non-positive & 74.23 \\
\hline +Inception-V2 Scene Model & 76.39 \\
\hline +SE-ResNet-50 Scene Model & 76.85 \\
\hline +SE-ResNet-50 Skeleton Model & 78.36 \\
\hline +Attention LSTM Model & 78.98 \\
\hline
\end{tabular}

further improve accuracy. The hybrid network is built by fusing the predictions ${ }^{4}$ from individual models. Exhaustive grid search is used to calculate the weights of the predictions of each model. The weights range from 0 to 1 , with increments of 0.05 and their sum is constrained to be 1 . The resulting weight of a redundant model is 0 .

Since face models have high accuracy and low false-positive rate on the positive class, the non-positive predictor, described in [11], is used in combination with the fine-tuned VGG-FACE and VGG2Senet-ft-Face models ${ }^{5}$ so that weights can be separately assigned to positive and non-positive classes during grid search. Table 2 shows the performance gain by adding one model at a time to previously fused models ${ }^{6}$.

\subsection{Submission Results}

The challenge allows 7 submissions in total. For the first submission, we trained models on the training data only and learned the

\footnotetext{
${ }^{4}$ By predictions we mean the probabilities of an image belonging to each class.

${ }^{5}$ Positive-only predictor was also applied, but the weight of it ended up being 0 after grid search.

${ }^{6}$ Note that due to the length limitations of the paper, redundant models have been removed and are not mentioned in this paper.
}

Table 3: Submission Results

\begin{tabular}{|c|c|c|c|}
\hline Sub & Training Data & Val & Test \\
\hline 1 & Training Set Only & 78.98 & 64.96 \\
\hline 2 & Training Set Only & 67.21 & 59.81 \\
\hline 3 & Training + Val & - & 64.73 \\
\hline 4 & Training + Val & - & 65.86 \\
\hline 5 & Training + Val & - & 67.59 \\
\hline 6 & Training + Val & - & 67.32 \\
\hline 7 & Training + Val & - & $\mathbf{6 8 . 0 8}$ \\
\hline
\end{tabular}

Table 4: Confusion matrix of submission 1, with overall accuracy being $64.96 \%$ on the testing data.

\begin{tabular}{|c|c|c|c|}
\hline & Neg & Neu & Pos \\
\hline Neg & 472 & 167 & 190 \\
\hline Neu & 147 & 510 & 259 \\
\hline Pos & 164 & 128 & 974 \\
\hline
\end{tabular}

Table 5: Confusion matrix of submission 7 , with overall accuracy being $68.08 \%$ on the testing data.

\begin{tabular}{|c|c|c|c|}
\hline & Neg & Neu & Pos \\
\hline Neg & 559 & 128 & 142 \\
\hline Neu & 156 & 529 & 231 \\
\hline Pos & 188 & 116 & 962 \\
\hline
\end{tabular}

weights of the decision fusion by favoring the highest accuracy on the validation data. Table 3 shows it overfits the testing set. The second submission is a single Inception-V2 model on scene. The 3-4 submissions and 5-7 submissions are trained on the combination of training and validation datasets with and without large-margin softmax loss, respectively. Confusion matrices of submission 1 and 7 are shown in Table 4 and Table 5, respectively.

\section{CONCLUSIONS}

In this paper, a hybrid network that combines 8 models for grouplevel emotion recognition in the wild is proposed. To the best of our knowledge, visual attention mechanism is presented and explored for the group emotion recognition problem for the first time in this paper. The overall accuracy of the proposed method, which scored the first place in the EmotiW Group-level Emotion Recognition Sub-challenge, is $68.08 \%$ on the test data.

\section{ACKNOWLEDGMENTS}

The work is supported by the National Science Foundation under Grant No. 1319598. 


\section{REFERENCES}

[1] Peter Anderson, Xiaodong He, Chris Buehler, Damien Teney, Mark Johnson, Stephen Gould, and Lei Zhang. 2017. Bottom-Up and Top-Down Attention for Image Captioning and VQA. CoRR abs/1707.07998 (2017). arXiv:1707.07998 http://arxiv.org/abs/1707.07998

[2] J. Bullington. 2005. Affective computing and emotion recognition systems: the future of biometric surveillance?. In Proceedings of the 2nd annual conference on Information security curriculum development. ACM, 95-99.

[3] Qiong Cao, Li Shen, Weidi Xie, Omkar M. Parkhi, and Andrew Zisserman 2017. VGGFace2: A dataset for recognising faces across pose and age. CoRR abs/1710.08092 (2017). arXiv:1710.08092 http://arxiv.org/abs/1710.08092

[4] Z. Cao, T. Simon, S. Wei, and Y. Sheikh. 2016. Realtime multi-person 2D pose estimation using part affinity fields. arXiv preprint arXiv:1611.08050 (2016).

[5] J. Deng, W. Dong, R. Socher, L.-J. Li, K. Li, and L. Fei-Fei. 2009. ImageNet: A Large-Scale Hierarchical Image Database. In CVPR.

[6] A. Dhall, A. Asthana, and R. Goecke. 2010. Facial expression based automatic album creation. In International Conference on Neural Information Processing. Springer, 485-492.

[7] A. Dhall, R. Goecke, and T. Gedeon. 2015. Automatic group happiness intensity analysis. IEEE Transactions on Affective Computing 6, 1 (2015), 13-26.

[8] A. Dhall, J. Joshi, K. Sikka, R. Goecke, and N. Sebe. 2015. The more the merrier: Analysing the affect of a group of people in images. In IEEE International Conference and Workshops on Automatic Face and Gesture Recognition, Vol. 1. IEEE, 1-8.

[9] Abhinav Dhall, Amanjot Kaur, Roland Goecke, and Tom Gedeon. 2018. EmotiW 2018: Audio-Video, Student Engagement and Group-Level Affect Prediction ( $A C M$ International Conference on Multimodal Interaction 2018 (in press)). ACM.

[10] I.J. Goodfellow et al. 2013. Challenges in representation learning: A report on three machine learning contests. In International Conference on Neural Information Processing. Springer, 117-124.

[11] X. Guo, L.F. Polanía, and K.E. Barner. 2017. Group-level emotion recognition using deep models on image scene, faces, and skeletons. In Proceedings of the 19th ACM International Conference on Multimodal Interaction. ACM, 603-608.

[12] Xin Guo, Luisa F. Polania, and Kenneth E. Barner. 2018. Smile detection in the wild based on transfer learning. (2018)

[13] Y. Guo, L. Zhang, Y. Hu, X. He, and J. Gao. 2016. MS-Celeb-1M: A Dataset and Benchmark for Large Scale Face Recognition. In European Conference on Computer Vision. Springer.

[14] K. He, X. Zhang, S. Ren, and J. Sun. 2016. Deep residual learning for image recognition. In CVPR. 770-778.

[15] S. Hochreiter and J. Schmidhuber. 1997. Long Short-Term Memory. Neural Comput. 9, 8 (Nov. 1997), 1735-1780.

[16] Jie Hu, Li Shen, and Gang Sun. 2017. Squeeze-and-Excitation Networks. CoRR abs/1709.01507 (2017). arXiv:1709.01507 http://arxiv.org/abs/1709.01507

[17] Xiaohua Huang, Abhinav Dhall, Guoying Zhao, Roland Goecke, and Matti PietikÃdinen. 2015. Riesz-based Volume Local Binary Pattern and A Novel Group Expression Model for Group Happiness Intensity Analysis. In BMVC. 1-9.

[18] A. Krizhevsky, I. Sutskever, and G.E. Hinton. 2012. Imagenet classification with deep convolutional neural networks. In Advances in Neural Information Processing Systems. 1097-1105.

[19] J. Li, S. Roy, J. Feng, and T. Sim. 2016. Happiness level prediction with sequential inputs via multiple regressions. In Proceedings of the 18th ACM International Conference on Multimodal Interaction. ACM, 487-493.

[20] Weiyang Liu, Yandong Wen, Zhiding Yu, and Meng Yang. 2016. Large-Margin Softmax Loss for Convolutional Neural Networks. In Proceedings of The 33rd
International Conference on Machine Learning. 507-516.

[21] Jiasen Lu, Caiming Xiong, Devi Parikh, and Richard Socher. 2017. Knowing When to Look: Adaptive Attention via A Visual Sentinel for Image Captioning.

[22] Volodymyr Mnih, Nicolas Heess, Alex Graves, and koray kavukcuoglu. 2014. Recurrent Models of Visual Attention. In Advances in Neural Information Processing Systems 27, Z. Ghahramani, M. Welling, C. Cortes, N. D. Lawrence, and K. Q. Weinberger (Eds.). Curran Associates, Inc., 2204-2212. http://papers.nips.cc/ paper/5542-recurrent-models-of-visual-attention.pdf

[23] W. Mou, O. Celiktutan, and H. Gunes. 2015. Group-level arousal and valence recognition in static images: Face, body and context. In IEEE International Conference and Workshops on Automatic Face and Gesture Recognition (FG), Vol. 5. IEEE, $1-6$.

[24] P.M. Niedenthal and M. Brauer. 2012. Social functionality of human emotion. Annual review of psychology 63 (2012), 259-285.

[25] O. M. Parkhi, A. Vedaldi, and A. Zisserman. 2015. Deep Face Recognition. In British Machine Vision Conference.

[26] F.E. Pollick, H.M. Paterson, A. Bruderlin, and A.J. Sanford. 2001. Perceiving affect from arm movement. Cognition 82, 2 (2001), B51-B61.

[27] Steven J. Rennie, Etienne Marcheret, Youssef Mroueh, Jarret Ross, and Vaibhava Goel. 2016. Self-critical Sequence Training for Image Captioning. CoRR abs/1612.00563 (2016). arXiv:1612.00563 http://arxiv.org/abs/1612.00563

[28] K. Simonyan and A. Zisserman. 2014. Very deep convolutional networks for large-scale image recognition. arXiv preprint arXiv:1409.1556 (2014).

[29] C. Szegedy, V. Vanhoucke, S. Ioffe, J. Shlens, and Z. Wojna. 2016. Rethinking the inception architecture for computer vision. In Proceedings of the IEEE Conference on Computer Vision and Pattern Recognition. 2818-2826.

[30] L. Tan, K. Zhang, K. Wang, X. Zeng, X. Peng, and Y. Qiao. 2017. Group emotion recognition with individual facial emotion $C N N$ s and global image based CNNs. In Proceedings of the 19th ACM International Conference on Multimodal Interaction. ACM, 549-552.

[31] S. Tomas, J. Hanbyul, M. Iain, and S. Yaser. 2017. Hand Keypoint Detection in Single Images using Multiview Bootstrapping. In CVPR.

[32] T. Vandal, D. McDuff, and R. El Kaliouby. 2015. Event detection: Ultra large-scale clustering of facial expressions. In IEEE International Conference and Workshops on Automatic Face and Gesture Recognition (FG), Vol. 1. IEEE, 1-8.

[33] Fei Wang, Mengqing Jiang, Chen Qian, Shuo Yang, Cheng Li, Honggang Zhang, Xiaogang Wang, and Xiaoou Tang. 2017. Residual Attention Network for Image Classification. CoRR abs/1704.06904 (2017). arXiv:1704.06904 http://arxiv.org/ abs/1704.06904

[34] S. Wei, V. Ramakrishna, T. Kanade, and Y. Sheikh. 2016. Convolutional pose machines. In $C V P R$

[35] J. Whitehill, G. Littlewort, I. Fasel, M. Bartlett, and J. Movellan. 2009. Toward practical smile detection. IEEE transactions on pattern analysis and machine intelligence 31, 11 (2009), 2106-2111.

[36] J. Wu and J.M. Rehg. 2011. CENTRIST: A Visual Descriptor for Scene Categorization. IEEE Trans. Pattern Anal. Mach. Intell. 33, 8 (2011), 1489-1501.

[37] Huijuan Xu and Kate Saenko. 2016. Ask, Attend and Answer: Exploring QuestionGuided Spatial Attention for Visual Question Answering. In Computer Vision - ECCV 2016 - 14th European Conference, Amsterdam, The Netherlands, October 11-14, 2016, Proceedings, Part VII. 451-466.

[38] Zichao Yang, Xiaodong He, Jianfeng Gao, Li Deng, and Alexander J. Smola. 2015. Stacked Attention Networks for Image Question Answering. CoRR abs/1511.02274 (2015). http://arxiv.org/abs/1511.02274

[39] K. Zhang, Z. Zhang, Z. Li, and Y. Qiao. 2016. Joint Face Detection and Alignment Using Multitask Cascaded Convolutional Networks. IEEE Signal Processing Letters 23, 10 (Oct 2016), 1499-1503. https://doi.org/10.1109/LSP.2016.2603342 